Preprint of an article published in Electrostatics to Photonics: Fundamentals and Applications for Physicists and Engineers. World Scientific, pp. 231-261. ISBN 9789813270268, 10.1142/9789813270268 0007 Copyright (C) 2020 by World Scientific Publishing Co. Pte. Ltd. https://www.worldscientific.com/worldscibooks/10.1142/10987$\underline{\text { voll }}$

Macedo, R. and Stamps, R. L. (2020) Electromagnetic waves in canted magnets. In: Donahue, M. (ed.) Compendium on Electromagnetic Analysis From Electrostatics to Photonics: Fundamentals and Applications for Physicists and Engineers. World Scientific, pp. 231-261. ISBN 9789813270268 (doi:10.1142/9789813270268_0007)

This is the Author Accepted Manuscript.

There may be differences between this version and the published version. You are advised to consult the publisher's version if you wish to cite from it.

http://eprints.gla.ac.uk/221990/

Deposited on: 11 August 2020

Enlighten - Research publications by members of the University of Glasgow http://eprints.gla.ac.uk 


\title{
Electromagnetic Waves in Canted Magnets
}

\author{
R. MACÊDO AND ROBERT L. STAMPS \\ University of Glasgow, Glasgow, United Kingdom G12 8QQ; University of \\ Manitoba, Winnipeg, MB Canada R3T 2N2 \\ August 11, 2020
}

\begin{abstract}
Summary
The optical properties of canted antiferromagnetics is discussed from the perspective of magnetic polaritons and field tunable electromagnetic properties. Canted antiferromagnets have a weak magnetization and their electromagnetic properties can be affected by applied magnetic and in the case of multiferroics, applied electric fields. A theoretical approach is outlined here that is useful for describing the $\mathrm{THz}$ frequency electromagnetic response and properties of a large variety of canted spin systems. The properties of two example systems are discussed: an antiferromagnet with canted induced through an externally applied magnetic field, and an antiferromagnetic multiferroic wherein the magnetic sublattices are canted by an internal coupling to a spontaneous dielectric polarisation.
\end{abstract}

Keywords: Magnetic polaritons, canted antiferromagnet, multiferroic, hyperbolic media

\section{Contents}

1 Introduction $\quad 2$

2 A Semi-classical Description of Polaritons 3

2.1 Spectra: Bulk modes and Reststrahl . . . . . . . . . . . 8

2.2 Reflection ..................... 11

2.2.1 Lateral Shifts and Nonreciprocity . . . . . . . . . . . 12

2.3 Refraction ..................... 15

2.3.1 Tunable Hyperbolic Dispersion and Focusing . . . . 16

2.4 Surface Modes and Attenuated Total Reflection . . . . . . . . 21

2.4.1 Trnasverse Electric (TE) Polarisation . . . . . . . . . . 21 
2.4.2 Transverse Magnetic (TM) Polarisation . . . . . . . . 25

3 Summary and Outlook 27

\section{Introduction}

The ability to generate strong electromagnetic waves in the $\mathrm{THz}$ region has motivated interest in using anisotropic media to access and manipulate electromagnetic waves in the millimeter and micrometer range [1]. However, the concept that the internal structure of a medium could affect its response to light has been known for centuries. A well known media with absorptiion resonances in this wavelength range are magnetic media such as antiferromagnets [2]. Propagating electromagnetic excitations of antiferromagnets are magnon-polaritons and these can be described by permeabilities which display sharp responses near resonances in the magnetic degrees of freedom [3]. These resonances can exist in the near infrared, and define bands of optical frequency transmission separated by restrahlen gaps whose width is strongly field dependent [4].

These antiferroagnets and electromagnetic properties associated with their resonances have been extesively studied since the early 50s [5]. The first structures to be investigated in this field were simple arrangements of spins perfectly aligned anti-parallel to one another and, therefore, posessing zero net magnetic moment $[3,6]$. Shortly after, a subclass of these structures gained in interest, that of 'canted antiferromagnets', in which a small feromagnetic component was seen along the direction of canting. This could be induced by simply applying an external magnetic field perpendicular to spin alignement direction. However, such behaviour has been also reported to happen naturally in a variety of magnetic crystals. Soviet literature, for instance, is resposiable for a great deal on the early investigations in this topic. The systems investigated include the orthorhombic crystal $\mathrm{NaNiF}_{3}$ [7] and $\alpha-\mathrm{Fe}_{2} \mathrm{O}_{3}$ [8]. Spontaneous canting was first explained by Dyzaloshinsky who showed that a weak ferromagnetism could be observed in the antiferromagnet $\alpha-\mathrm{Fe}_{2} \mathrm{O}_{3}$ and other materials with a symmetry breaking effective field arising from spin orbit coupling effects on electronic exchange interactions. These fields serve to turn the antiferromagnetically coupled sublattices towards each other, producing a net magnetic moment, and is often used in models of multiferroic response of magnetic degrees of freedom to electric fields [9].

In this chapter a theoretical description is presented for electromagnetic wave propagation in canted antiferromagnets. Two examples are discussed. 
In the first example, the spin canting is achieved by application of a magnetic field perpendicular to the unaxial anisotropy axis of a colinear antiferromagnet. In the second example, the canting appears as a spontaneous deformation of a colinear antiferromagnet due to the generation of a electric polarisation via a Dyzaloshinskii mechaism. Through these examples, properties of bulk and surface magnetic polaritons in antiferromagnets are described, and interesting consequences for reflection and transmission in film geometries are discussed.

\section{A Semi-classical Description of Polaritons}

In order to discuss optical properties in canted antiferromagnets, and ultimatly, the effect of magneto-electric coupling in spin systems, it is useful to summarize the general behaviour of polaritons in the context of electromagnetic phenomenology.

When electromagnetic waves interact with condensed matter they can excite the material's internal degrees of freedom. The propagation of electromagnetic waves in the material will be modified strongly near absorption resonances associated with these excitations. This can appear in the dispersive characteristics of the electromagnetic wave as a deviation from the ligth line in a material that is most pronounced near material resonances [10]. These phenomena are described with constitutive relations that couple the displacement $\mathbf{D}$ and induction $\mathbf{B}$ fields with the medium through linear response permittivity $\stackrel{\leftrightarrow}{\varepsilon}(\omega)$ and permeability $\overleftrightarrow{\mu}(\omega)$ [1]. The general form of the constitutive relations is

$$
\mathbf{D}=\overleftrightarrow{\varepsilon}(\omega) \mathbf{E}
$$

and

$$
\mathbf{B}=\overleftrightarrow{\mu}(\omega) \mathbf{H}
$$

The nature of these resonances depends on their origin. Electromagnetic coupling to elastic properties of a material can generate optical frequency lattice vibrations which are referred to as phonon-polaritons [10]. The simplest situation is that in which the crystal has a single infrared active phonon-polariton so that the dielectric constant is frequency dependent and can be described by [11]

$$
\varepsilon(\omega)=\varepsilon_{\infty}+\frac{\Omega^{2}}{\omega_{T O}^{2}-\omega^{2}} .
$$


Here $\omega_{T O}$ is the transverse-optical phonon frequency, $\Omega$ its oscillator strength and $\varepsilon_{\infty}$ the contributions to the dielectric constant from high frequency excitations.

In a variety of crystals, however, phonon-polaritons have been reported to be anisotropic i.e., propagation depends on direction relative to the symmetry axes of the crystal. This means that the dielectric function differs for different directions and is expressed in a tensorial form [1]. In this case, if the Cartesian axes can be chosen to align with the principal axes of the crystal, the tensor becomes diagonal and can be written as follows

$$
\overleftrightarrow{\varepsilon}(\omega)=\left(\begin{array}{ccc}
\varepsilon_{x x} & 0 & 0 \\
0 & \varepsilon_{y y} & 0 \\
0 & 0 & \varepsilon_{z z}
\end{array}\right) .
$$

Similarly to dielectric crystals interacting with the electric field of radiation, magnetic media respond to dynamic magnetic fields. In this case, The response is described by the magnetic permeability [10, 12]. In antiferromagnets, for instance, the magnetic field of an electromagnetic wave excites spin precession. The frequency at which the radiation couples with the spin precession is given by

$$
\omega_{0}= \pm \gamma\left(2 B_{A} B_{E}+B_{A}^{2}\right)^{1 / 2} .
$$

where $B_{A}$ and $B_{E}$ are the internal anisotropy and exchange fields acting on the spins and keeping them aligned anti-parallel to one another [3, 13]. This resonance determines the frequency of a singularity in the magnetic permeability. In a colinear antiferromagnet, the magnetic sublattices are aligned anti-parallel to one another. Denoting these orientations as $+y$ and $-y$, the precession and corresponding linear response is in the transverse $x$ and $z$ directions. This means that $\mu_{y y}$ is unity at all frequencies and the other components are given by

$$
\mu_{x x}=\mu_{z z}=1+\frac{2 \mu_{0} \gamma^{2} M_{S} B_{A}}{\omega_{0}^{2}-\omega^{2}} .
$$

Similarly to the case of Eq. (4) for phonon-polaritons in dielectric media, the linear response of antiferromagnets to a time varying magnetic field can be represented through the permeability tensor:

$$
\overleftrightarrow{\mu}(\omega)=\left(\begin{array}{ccc}
\mu_{x x} & 0 & 0 \\
0 & \mu_{y y} & 0 \\
0 & 0 & \mu_{z z}
\end{array}\right) .
$$

Though diagonal, the susceptibilities $\overleftrightarrow{\varepsilon}(\omega)$ and $\overleftrightarrow{\mu}(\omega)$ are examples of anisotropic media. In the following sections, we consider off-diagonal 
elements in the susceptibilities and discuss examples of how anisotropic magnetic media with canted spins can be used to induce unusual optical effects on reflection, transmission and surface waves. The off-diagonal elements will describe weak magnetism. In the first example the off diagonal elements appear in the permeability and are created by an externally applied magnetic field. In the second example the weak magnetism is created spontaneously through an effective internal field that describes coupling to the dielectric properties of the material and is expressed in an additional magneto-electric susceptibility that in principle involves the time varying electric and time varying magnetic fields.

\section{Polaritons in Field-Canted Antiferromagnets}

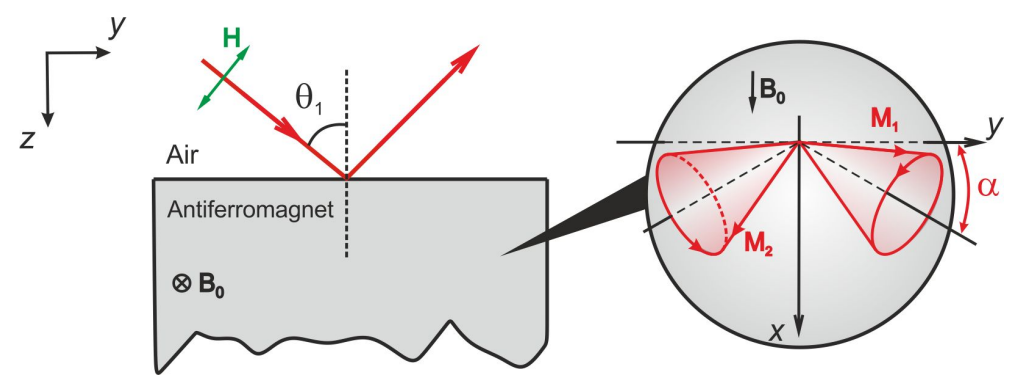

Figure 1: Schematics of an electromagnetic beam incidence at the surface of an antiferromagnet as considered throughout this section. The plane of incidence is the $y z$-plane and the radiation is considered to be transverse electric (TE) polarized so that the time-varying $\mathbf{H}$ field of the incident beam lies in the $y z$-plane. In the absence of an external field $\mathbf{B}_{0}$ the sublattice magnetisation, as well as the anisotropy field, are parallel to the surface and directed along $+y$ and $-y$. In the case of a nonzero external field $\mathbf{B}_{\mathbf{0}}$ applied along $x$, and perpendicular to the incidence plane, the spins cant by an angle $\alpha$ towards the applied field direction as shown in the inset. For clarity only, the vertical axes in the inset have been flipped.

As discussed earlier, we will start our discussion by looking at a canted spin system due to a static Zeeman field externally applied, such as the cartoon in the inset of Fig. 1. This sufficiently describes classic collinear antiferromagnets, such as the salts $\mathrm{MnF}_{2}, \mathrm{FeF}_{2}$ and $\mathrm{CoF}_{2}$ [10]. In this case, the minimization of the competing anisotropy $\mathbf{B}_{\mathbf{A}}$ and exchange $\mathbf{B}_{\mathrm{E}}$ fields leads to a ground state of spins perfectly aligned antiparallel to one another. However, spin canting can be induced by simply applying an external 
magnetic field $\mathbf{B}_{0}$ perpendicular to the spin alignment direction as shown in Fig. 1. Precession motion of the spins is driven by a time-varying $\mathbf{h}(t)$ of frequency $\omega$ given by

$$
\mathbf{h}(t)=\left(\hat{x} h_{x}+\hat{y} h_{y}+\hat{z} h_{z}\right) e^{-j \omega t} .
$$

Here we consider $\mathbf{M}_{\mathbf{1}}$ and $\mathbf{M}_{\mathbf{2}}$ as the magnetization of each sublattice. In the Cartesian coordinate system we have: the static,external Zeeman field $\mathbf{B}_{\mathbf{0}}$ is parallel to the $x$ axis; we take both $\mathbf{M}_{\mathbf{1}}$ and $\mathbf{M}_{\mathbf{2}}$ to lie in the $y$ axis, in their equilibrium configuration. Thus, the effective fields, $\mathbf{H}_{\mathbf{e f f}}^{(\mathbf{i})}$, acting on a sublattice $i$ is given by $[14,15,16]$

$$
\mathbf{H}_{\mathrm{eff}}^{(\mathbf{i})}=\frac{1}{\mu_{0}}\left(\mathbf{B}_{\mathbf{i A}}+\mathbf{B}_{\mathbf{i E}}+\mathbf{B}_{\mathbf{0}}\right)+\mathbf{h}(t) .
$$

The external field, $\mathbf{B}_{0}$, leads to a spin canting of magnitude $\alpha$ and we can write expressions for the magnetization of each sublattice as

$$
\mathbf{M}_{\mathbf{1}}=M_{s}(\hat{x} \sin \alpha+\hat{y} \cos \alpha)+\mathbf{m}^{(\mathbf{1})}
$$

and

$$
\mathbf{M}_{\mathbf{2}}=M_{s}(\hat{x} \sin \alpha-\hat{y} \cos \alpha)+\mathbf{m}^{(2)}
$$

where $M_{S}$ is the static magnetization and $\mathbf{m}^{(\mathbf{1})}, \mathbf{m}^{(\mathbf{2})}$ are the time-dependent components associated with the spin wave in the structure. We can then write classic equations of motion of the various spin components which yield coupled equations for $\mathbf{m}_{\mathbf{1}}$ and $\mathbf{m}_{\mathbf{2}}$. These equations can be written through

$$
\frac{1}{\gamma} \frac{d \mathbf{M}_{\mathbf{i}}}{d t}=\mu_{0} \mathbf{M}_{\mathbf{i}} \times \mathbf{H}_{\mathrm{eff}}^{(\mathbf{i})}
$$

This yields six coupled equations for each component of the two sublattice magnetizations. Ultimately, we assume that the $\mathbf{m}^{(\mathbf{i})}$ quantities are small and we can linearize the equations of motion by ignoring quadratic dynamic terms.

There are two main properties of the spin system which we are interested here and that can be obtained from the equations of motion:

1. We can use these equations to find the resonances in the canted antiferromagnets. In our particular case, the time-varying fields disturbs the spin system, generating resonant behaviour in all directions. This means that there are more than one resonance. These can be found by setting $h=0$ and subsequently writing all components of the dynamic magnetization as a matrix of the form: 


$$
\Omega\left(\begin{array}{l}
m_{x}^{(1)} \\
m_{y}^{(1)} \\
m_{z}^{(1)} \\
m_{x}^{(2)} \\
m_{y}^{(2)} \\
m_{z}^{(2)}
\end{array}\right)=0 .
$$

Thus, the resonance frequencies can be obtained from the secular determinant of $\Omega$. Given in terms of $\omega_{0}$, they assume the form

$$
\omega_{\|}^{2}=\omega_{0}^{2} \cos ^{2} \alpha
$$

and

$$
\omega_{\perp}^{2}=\omega_{0}^{2} \cos ^{2} \alpha+2 B_{E} B_{0} \sin \alpha .
$$

These resonances have a clear dependence on the canting angle $\alpha$, and hence external field $\mathbf{B}_{\mathbf{0}}$. This angle can be found by finding the direction of equilibrium of the magnetization, also from the same set of equations, and can be written as:

$$
\sin \alpha=\frac{B_{0}}{B_{A}+2 B_{E}} .
$$

2. We can also use these equations to rewrite the permeability tensor and its components, given by Eq. 7, as to account for the spin canting. This time, we need to assume a non-zero time varying external field so that the equations of motion may be written as:

$$
m_{i}=\stackrel{\leftrightarrow}{\chi}_{i j}(\omega) h_{j}
$$

where $\overleftrightarrow{\chi}_{i j}(\omega)$ relates the alternating magnetization to the time varying magnetic field, it may be called the high frequency susceptibility. To convert this into the canonical permeability tensor we use the identity $\overleftrightarrow{\mu}(\omega)=\delta_{i j} \overleftrightarrow{\chi}(\omega)$, where $\delta_{i j}$ is the identity matrix. In a tensor form, Eq. 7 now assumes the form

$$
\overleftrightarrow{\mu}(\omega)=\left(\begin{array}{ccc}
\mu_{x x} & 0 & 0 \\
0 & \mu_{y y} & -j \mu_{y z} \\
0 & j \mu_{y z} & \mu_{z z}
\end{array}\right)
$$


with components written as now written as

$$
\begin{gathered}
\mu_{x x}=1+\frac{2 \mu_{0} \gamma^{2} M_{S} B_{E} \cos ^{2} \alpha}{\omega_{\|}^{2}-\omega^{2}}, \\
\mu_{y y}=1+\frac{2 \mu_{0} \gamma^{2} M_{S} B_{0} \sin \alpha}{\omega_{\perp}^{2}-\omega^{2}}, \\
\mu_{z z}=1+\frac{2 \mu_{0} \gamma^{2} M_{S}\left(B_{0} \sin \alpha+B_{A} \cos 2 \alpha\right)}{\omega_{\perp}^{2}-\omega^{2}},
\end{gathered}
$$

while

$$
\mu_{y z}=-\mu_{y z}=-j \frac{2 \mu_{0} \gamma^{2} M_{S} \omega \sin \alpha}{\omega_{\perp}^{2}-\omega^{2}} .
$$

Note that in this plane, the precession direction is the same for the two sublattices, but changes direction when the field direction is reversed. The antiferromagnet is thus gyromagnetic, with nonzero permeability components $\mu_{y z}$ and $\mu_{z y}$ whose signs depend on the field direction [15]. A full description of these and the methods of how they can be derived from the equations of motion has been detailed in Chapter 1 by Anderson, Camley and Livesey.

\subsection{Spectra: Bulk modes and Reststrahl}

The dynamic response of antiferromagnets to optical waves is thus determined by competition between components of the permeability tensor. Having now discussed the nature of resonances and general structure of the tensor in a field canted spin system, we now turn to the electromagnetic problem. This begins with Maxwell's equations. In the present case, where Maxwell's equations without sources or currents hold, we have:

$$
\begin{gathered}
\nabla \cdot \mathbf{D}=0, \\
\nabla \times \mathbf{E}=-\frac{\partial \mathbf{B}}{\partial t}, \\
\nabla \cdot \mathbf{B}=0, \\
\nabla \times \mathbf{H}=\frac{\partial \mathbf{D}}{\partial t}
\end{gathered}
$$

We consider the electromagnetic waves to be propagating from vacuum onto a uniaxial antiferromagnet in the geometry shown in Fig. 1 with 
incidence plane $y z$. If the angle of incidence is represented as $\theta_{1}$ and $k_{0}=$ $\omega / c$, the in-plane wave vector component $k_{y}$ is the same across all layers and is given by

$$
k_{y}=k_{0} \sin \theta_{1} .
$$

The $z$ component of the wave vector, however, assumes different forms through the different layers. In this case, it is useful to employ Maxwell's equations in the two media. This can be done by combining Eqs. (23b) and (23d) to find the wave equations for both $\mathbf{E}$ and $\mathbf{H}$. These can be written as

$$
i k \times\left[\stackrel{\leftrightarrow}{\varepsilon}(\omega)^{-1}(i k \times \mathbf{H})\right]=\frac{\omega^{2}}{c^{2}} \overleftrightarrow{\mu}(\omega) \mathbf{H}
$$

and

$$
i k \times\left[\overleftrightarrow{\mu}(\omega)^{-1}(i k \times \mathbf{E})\right]=-\frac{\omega^{2}}{c^{2}} \overleftrightarrow{\varepsilon}(\omega) \mathbf{E} .
$$

These equations are symmetric and can be interchanged by one another. For simplicity, we will carry on working only with $\mathbf{H}$.

In vacuum, we can then use Eq. (25) to find the wavevector component $k_{z 1}$ as being

$$
k_{z 1}^{2}=k_{0}^{2}-k_{y}^{2} .
$$

In the antiferromagnetic crystal, on the other hand, the properties of the wave vector component $k_{z 2}$ depend on the polarization of the propagating wave. In the case of TE polarised waves, this component is given by

$$
k_{z 2}^{2}=\frac{\varepsilon k_{0}^{2}\left(\mu_{y y} \mu_{z z}-\mu_{y z}^{2}\right)-\mu_{y y} k_{y}^{2}}{\mu_{z z}},
$$

while for TM polarised waves we have

$$
k_{z 2}^{2}=\frac{\mu_{x x}}{\varepsilon} k_{0}^{2}-k_{y}^{2} .
$$

These equations can be, in a way, regarded as a generalised Fresnel's equation of the wave normals for a field canted antiferromagnet.

In order to illustrate the nature of magnon-polaritons in antiferromagnets the following sections use the example antiferromagnet $\mathrm{MnF}_{2}$. This is a two-sublattice uniaxial antiferromagnet of simple structure which has been extensively investigated theoretically as well as experimentally. For this material, the relevant parameters [17] at a temperature of $4.2 \mathrm{~K}$ are $\mathrm{M}_{S}=$ $6.0 \times 10^{5} \mathrm{~A} / \mathrm{m}$ and $\mathrm{B}_{A}=0.787 \mathrm{~T}$. The Néel temperature is $67 \mathrm{~K}$ and the corresponding exchange field is $B_{E}=53.0 \mathrm{~T}$. Also $\gamma / 2 \pi c=0.975 \mathrm{~cm}^{1} / \mathrm{T}$, 


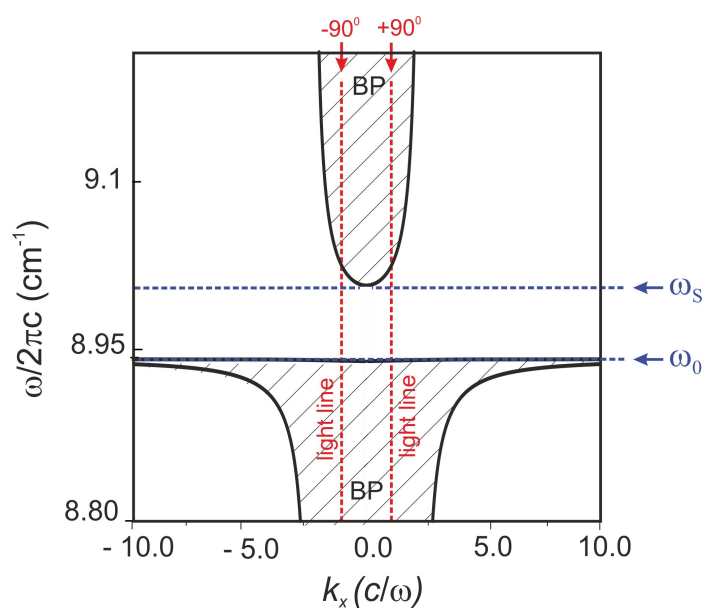

Figure 2: Magnon-polariton dispersion relation for TM-polarized radiation incident on a $\mathrm{MnF}_{2}$ surface for $B_{0}=0$. The shaded regions represent propagation inside the material while no propagation is shown elsewhere. Based on data from R. Macêdo. Tunable Hyperbolic Media: Magnon-Polaritons in Canted Antiferromagnets. In Robert E. Camley and Robert L. Stamps: Solid State Physics, 68, 91-155 (2017).

corresponding to $\omega_{0} / 2 \pi c=8.94 \mathrm{~cm}^{-1}$. The damping parameter is $\Gamma / 2 \pi c$ $=0.0007 \mathrm{~cm}^{-1}$. In the case where $\mathrm{B}_{0}=0, \mu_{z z}$ has a pole at the resonance frequency $\omega_{0} / 2 \pi c=8.94 \mathrm{~cm}^{-1}$ and a zero at a somewhat higher frequency close to $9.00 \mathrm{~cm}^{-1}$. Even though it has been pointed out that the dielectric constant in $\mathrm{MnF}_{2}$ is also anisotropic and should be treat in the form given by Eq. (4) [18], we simply treat this quantity as isotropic $\varepsilon=5.5$. This does not affect the nature of the effects we will discuss and this approach has been shown to be consistent with experimental works in this spectral area [17].

In order to illustrate the TM polarised wave propagation, we show the plotted dispersion relation in Fig. 2. Because Eq. 29 only couples with the resonant component $\mu_{x x}$ which the negative between $\omega_{\|}$and $\omega_{S}$, no propagation is allowed in this region. This is called the reststrahl region. The areas of propagation as shown as two shaded regions, the upper and lower bulk polariton bands(BP).

The most interesting case, however, is that of TE polarised incident waves shown in Fig. 3. In this plane of polarisation, and in the absence of an external field as shown in (a), propagation is possible for every incident $k_{y}$ in the frequency range between $\omega_{0}$ and $\omega_{S}$. In contrast to the case of TM polarised waves, there is no reststrahl gap between the upper and lower bulk bands at $k_{y}=0$. This is because in this geometry, the magnetic field 


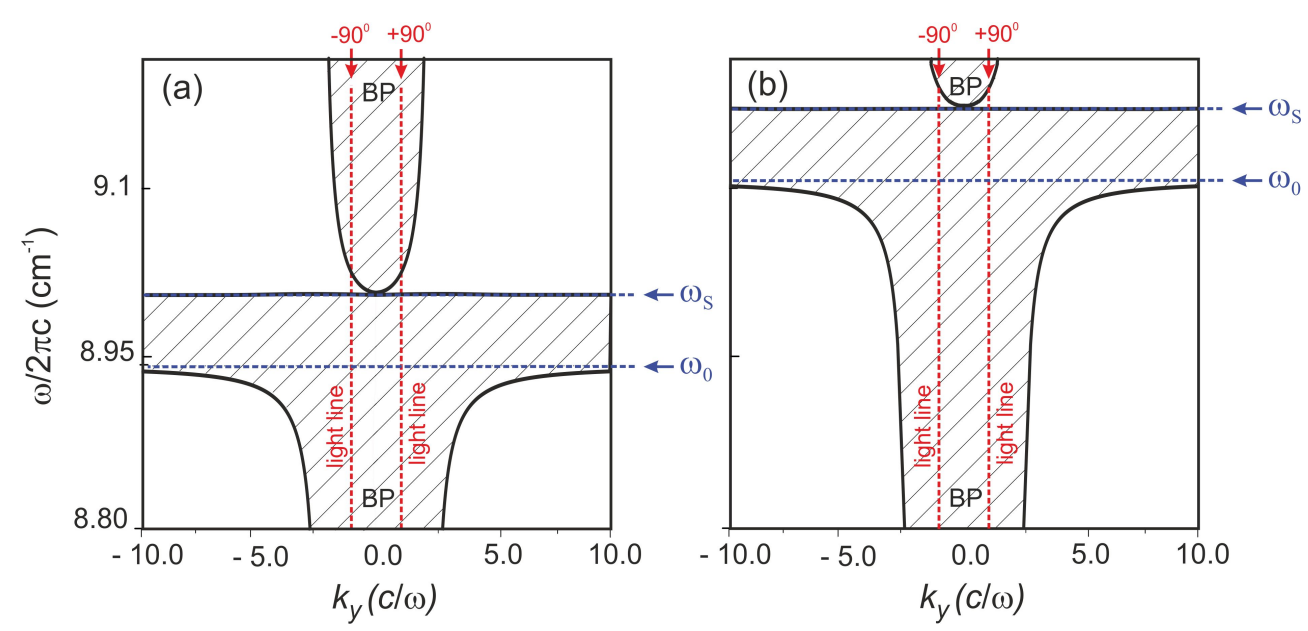

Figure 3: Magnon-polariton dispersion relation for TM-polarized radiation incident on a $\mathrm{MnF}_{2}$ surface. Here (a) is for $B_{0}=0$ and (b) is for $B_{0}=1.5$ $\mathrm{T}$. The shaded regions represent propagation inside the material while no propagation is shown elsewhere. Based on data from R. Macêdo. Tunable Hyperbolic Media: Magnon-Polaritons in Canted Antiferromagnets. In Robert E. Camley and Robert L. Stamps: Solid State Physics, 68, 91-155 (2017).

of light couple with two different permeability tensor components with opposing signs.

The nature of this behaviour is not affected by an external field as shown in Fig. 3(b). However, the field does shifts the resonance to higher frequency and thus, shifting the features of the dispersion relationr. Another consequence of the off-diagonal components $\mu_{y z}$ and $\mu_{z y}$ is to slightly separate the upper and lower bulk bands so that there is a small reststrahl gap just after $\omega_{S}$, even at $k_{y}=0$.

\subsection{Reflection}

In order to investigate further the behaviour of the dispersion relation shown previously, we look at the properties of a reflected beam from an antiferromagnetic surface. Here, we concentrate on a geometry in which the electric field of the propagating electromagnetic waves is polarised perpendicular to the plane of incidence (TE-polarised) and along $x$ as shown in FIg. 1. The reflectivity coefficient can be found by matching the boundary conditions in the surface $(z=0)$. The boundary conditions require the continuity of the tangential components of $\mathbf{E}$ and $\mathbf{H}$. This leads to a complex 
reflection coefficient $r$, written in terms of Eq. 18 as [15]

$$
r=\frac{E_{r x}}{E_{i x}}=\frac{k_{z 1}\left(\mu_{y y} \mu_{z z}+\mu_{y z}^{2}\right)-k_{z 2} \mu_{z z}-k_{y} \mu_{y z}}{k_{z 1}\left(\mu_{y y} \mu_{z z}+\mu_{y z}^{2}\right)+k_{z 2} \mu_{z z}+k_{y} \mu_{y z}}
$$

Note that $r$ is given in terms of the components of $\overleftrightarrow{\mu}(\omega)$, including the imaginary off-diagonal components $\mu_{y z}$ and $\mu_{z y}$. As discussed previously, the signs of these components depend on the field direction, and hence canting. If we then consider an steady field, so that the system is canted and these components always exist, their sign will simply depend on the magnetic field of the incident radiation. In the present case, the incident magnetic field has a component in the $y z$ plane, therefore, nonreciprocal effects may be expected in reflection and any other effect dependent on it.

\subsubsection{Lateral Shifts and Nonreciprocity}

In Fig. 4 we show the spectral reflectance $\left(R=r r^{*}\right)$ at the surface of an antiferromagnetic crystal for an incidence angles of $\pm 60^{\circ}$. We start by looking at the case where case when $\Gamma=0$ so that $k_{z 2}$ is either wholly real or imaginary. In this particular case, a real $k_{z 2}$ leads to propagation into the material, whereas an imaginary $k_{z 2}$ gives no propagation. The propagating and non-propagating regions are depicted in Fig. 3(b) as shaded and unshaded regions, respectively. Note that the shading regions are made to match those of Fig. 4. Another consequence of $\Gamma=0$ is that of only one line to be seen for either directions of the incident beam $\left( \pm 60^{\circ}\right)$, in which case $R$ is always unity when $k_{z 2}$ is imaginary and smaller elsewhere.

One important aspect of reflection in antferromagnetic crystals, however, is that of nonreciprocity when damping is included $(\Gamma \neq 0)$ [2]. This behaviour, has been explained in a simple manner by using symmetry arguments [19]. This dictates that when a spin system of this sort is subjected to an external magnetic field $\mathbf{B}_{\mathbf{0}}$ the symmetry of the system is broken so that

$$
R\left(\theta_{1}, \mathbf{B}_{\mathbf{0}}\right) \neq R\left(\theta_{1}, \mathbf{B}_{\mathbf{0}}\right) \neq R\left(\theta_{1},-\mathbf{B}_{\mathbf{0}}\right) .
$$

The nonreciprocal behaviour is shown by the solid lines in Fig. 4. It is clear from those that the different directions of propagation of the incident beam yield a distinct reflectance spectrum [20].

If we now consider this reflection to be that of a finite beam, we can model it to be a sum of plane waves. This spectrum approach has been used previously by McGuirk and Carniglia where the complex reflection coefficient is given by 

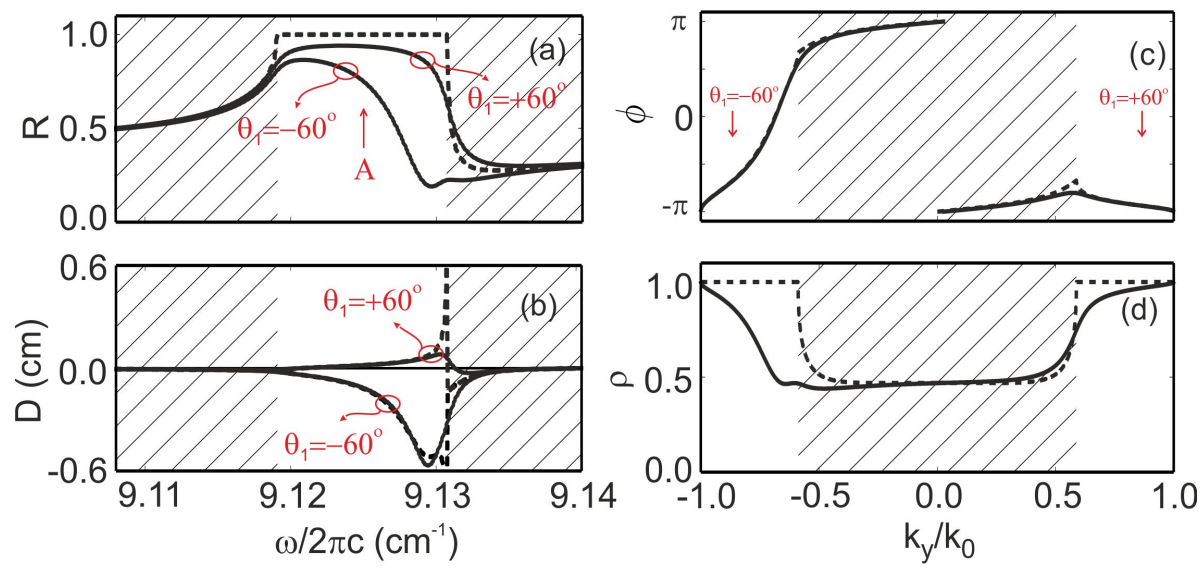

Figure 4: (a) Calculations of TE polarised oblique incidence $\left(\theta_{1}= \pm 60^{\circ}\right)$ reflection from the interface between vacuum and $\mathrm{MnF}_{2}$ in the presence of an external magnetic field of $1.5 \mathrm{~T}$ and (b) Goos-Hänchen shift D. Reflected (c) phase and (d) amplitude, as a function of in-plane wavevector $k_{y}$, at frequency marked as A $\left(9.125 \mathrm{~cm}^{-1}\right)$ in (a). Dashed lines are calculated ignoring damping, whereas solid lines are for calculations in which damping is included. The shaded region shows frequencies where transmission can occur in the absence of damping according to Fig. 3(b). Adapted from $R$. Macêdo, R. L. Stamps, and T. Dumelow, Spin canting induced nonreciprocal Goos-Hüchen shifts, Opt. Express 22, 28467 (2014).

$$
r\left(k_{y}\right)=\rho\left(k_{y}\right) e^{j \phi\left(k_{y}\right)}
$$

where $\rho\left(k_{y}\right)$ is the reflection amplitude and $\phi\left(k_{y}\right)$ is the associated phase change on reflection [21]. These are shown in Fig. 4(c) and (d), where can be seen that the phase is not always 0 or $\pi$. In this case, as the phase change varies with $k_{y}$, interference between the reflected plane waves will be different from that for the incident waves, leading to a change in the reflected beam profile. This typically manifests itself as a lateral shift of the reflected beam given by

$$
D=-\frac{d \phi}{d k_{y}}
$$

We depict this behaviour in Fig. 4(b) we show the corresponding values of this displacement. This is commonly known as Goos-Hänchen shifts, and in antiferromagnetic case, It can be immediately noticed that these are distinctly nonreciprocal $D\left(+\theta_{1}\right) \neq D\left(\theta_{1}\right)$, either with or without damping. 
When investigating lateral shifts of the reflected beam, it is also useful to consider energy flow within the crystal. This is of interest as it was used as an argument in theories, such as that of Renard [22] to described the behaviour of a displaced reflected beam. This is also particularly useful to directly visualised the behaviour of the displacement. In order to do this, we make used of the time-averaged Poynting vector $\left\langle\mathbf{S}_{\mathbf{2}}\right\rangle=1 / 2 \operatorname{Re}\left(\mathbf{E} \times \mathbf{H}^{*}\right)$. When considering a TE polarised wave, incident in an antiferromagnetic surface in the geometry shown in Fig. $1,\left\langle\mathbf{S}_{\mathbf{2}}\right\rangle$ has components

$$
\left\langle S_{2 y}\right\rangle=\frac{\left|E_{x}\right|^{2}}{2 \omega \mu_{0}} \operatorname{Re}\left(\frac{k_{y} \mu_{y y}-k_{2 z} \mu_{y z}}{\mu_{y y} \mu_{z z}+\mu_{y z}^{2}}\right)
$$

and

$$
\left\langle S_{2 z}\right\rangle=\frac{\left|E_{x}\right|^{2}}{2 \omega \mu_{0}} \operatorname{Re}\left(\frac{k_{2 z} \mu_{z z}+k_{y} \mu_{y z}}{\mu_{y y} \mu_{z z}+\mu_{y z}^{2}}\right) .
$$
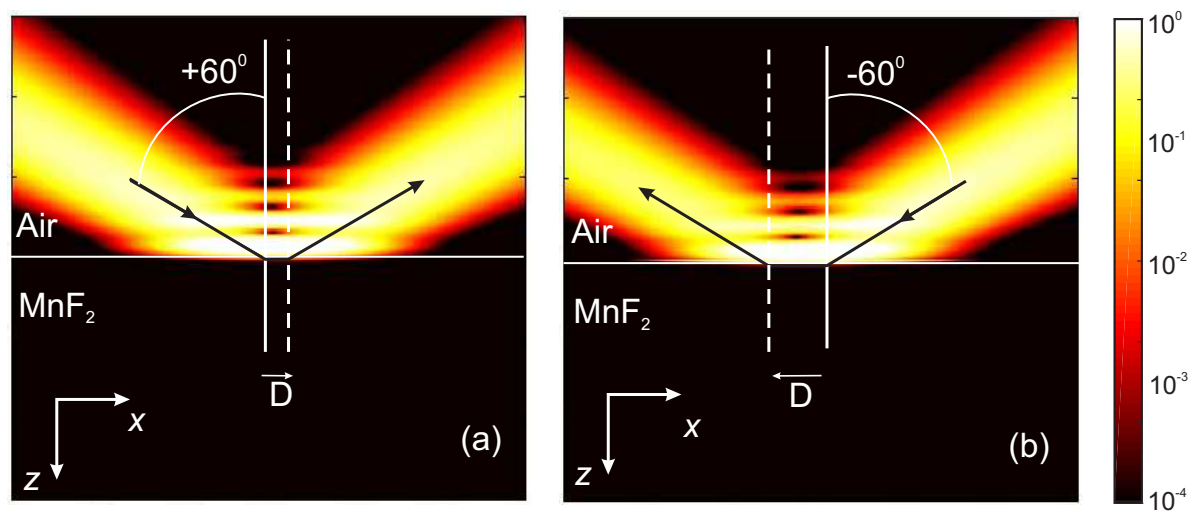

Figure 5: Calculated overall intensity of the magnitude of the time-averaged Poynting vector for a beam of $0.2 \mathrm{~cm}$ wide obliquely incident on a Air $/ \mathrm{MnF}_{2}$ interface at frequency $\mathrm{A}$ in the presence of a magnetic field $\mathrm{B}_{0}=1.5 \mathrm{~T}$. (a) $\theta_{1}=+60^{\circ}$ and (b) $\theta_{1}=-60^{\circ}$. The arrows represent the incident and reflected beams, positioned according to Eq. (33), with angle of reflection assumed equal to angle of incidence. Based on data from R. Macêdo, R. L. Stamps, and T. Dumelow, Spin canting induced nonreciprocal Goos-Hüchen shifts, Opt. Express 22, 28467 (2014).

The lateral displacement of a Gaussian beam incident on a $\mathrm{MnF}_{2}$ surface is shown in Fig. 5. The simulations shown use a plane wave spectrum model [21, 20], of a Gaussian beam of width $g=0.2 \mathrm{~cm}$ incident on the surface of a semi-infinite antiferromagnet. In Fig. 5(a), results are given for 
a positive incident angle corresponding to a small displacement as expected from Fig. 4(b). In Fig. 5(b) we show the case for a negative incident angle, with a much larger displacement also agreeing with the results shown in Fig. 4(b). Both cases cases shown in Fig. 5 were calculated for the same frequency masked as A in Fig. 4(a). This clearly depicts not only the lateral displacement, but also its nonreciprocal nature. Note that the nonreciprocity shown here is given in terms of the incident angle, but it would also be present by reversing the direction of the external field $\mathbf{B}_{0}$ due to the nature of the off-diagonal components of the permeability tensor, as discussed previously.

\subsection{Refraction}

Now that we have analysed spectral region of nonreciprocal reflection and the behaviour of the reflected beam, we now turn to the problem of the refracted beam. This is of particular interest as it has been recently reported that canted antiferromagnets can be used as a route to tunable negative refraction $[14,23]$. Here we take the angle of refraction $\theta_{2}$ to be given in terms of the Poynting vector $\boldsymbol{S}_{\mathbf{2}}$ inside the antiferromagnet. By the geometry shown in Fig. 1 we get

$$
\tan \theta_{2}=\frac{\left\langle S_{2 y}\right\rangle}{\left\langle S_{2 z}\right\rangle}
$$

It is easier to interpret the behaviour of the refracted by by starting with the situation in which there is no damping nor external applied field. In this case, $\mu_{y z}$ and $\mu_{z y}$ vanish and in the region between $\omega_{0}$ and $\omega_{S}, \mu_{y y}>1$ and $\mu_{z z}<1$. In this region $k_{2 z}$ is wholly real so that if we substitute this quantities into Eq. (36), is easy to see that $\theta_{1}$ and $\theta_{2}$ will always have opposing signs [24].

This can be visualised by simulating the behaviour of a Gaussian beam travelling through an antiferromagnetic slab as done in Sec. 2.2. This is shown in Fig. 6(a) where negative refraction of the power flow for a beam incident on a $\mathrm{MnF}_{2}$ slab is seen.

Because we are interested in the canted spin systems, we show the behaviour of the refracted beam for various fields in Figs. 6(b)-(c). When an nonzero external field is applied the values of the permeability tensor components change and so does the the magnitude of $\theta_{2}$. For the field values chosen, negative refraction of a Gaussian beam can be directly observed in all cases. However, a more complete analysis of this behaviour has been given by Macêdo and Dumelow showing that is possible to turn the negative refraction on and off according to the field strenght [14]. 

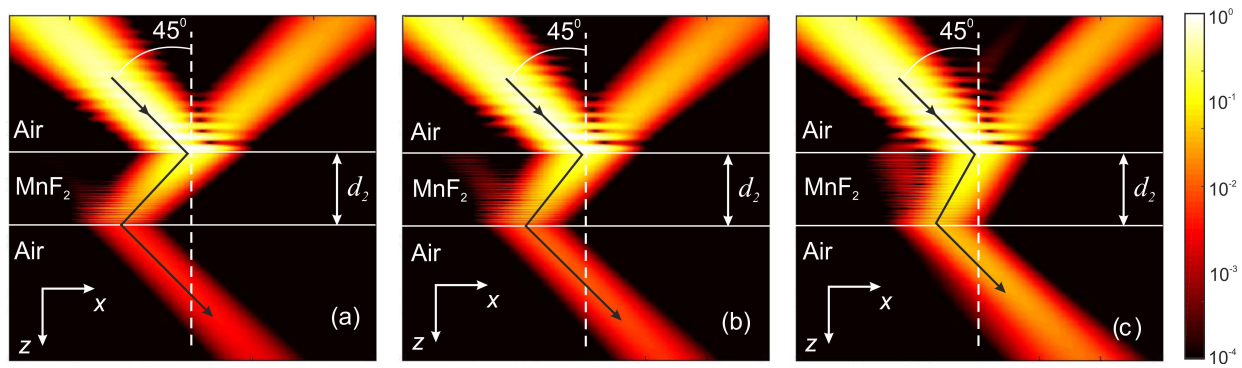

Figure 6: Intensity profile (time averaged power density) of a Gaussian beam obliquely incident at an angle of incidence $\theta_{1}=45^{0}$, passing through an $\mathrm{MnF}_{2}$ slab at frequency $\omega / 2 \pi c=8.984 \mathrm{~cm}^{-1}$. (a) $B_{0}=0.0$, (b) $B_{0}=0.2 \mathrm{~T}$ and (c) $B_{0}=0.4 \mathrm{~T}$. The intensity scale is in arbitrary units. Adapted from $R$. Macêdo, T. Dumelow, and R. L. Stamps, Tunable Focusing in Natural Hyperbolic Magnetic Media, ACS Photonics 3, 1670 (2016).

\subsubsection{Tunable Hyperbolic Dispersion and Focusing}

Even though we have just seen how negative refraction in canted antiferromagnets can be explained in terms of the power flow, this is commonly treated as hyperbolic behaviour [25]. This has been extensively investigated in context of metamaterials [26] and even natural dielectric crystals [27, 28]. The hyperbolic behaviour has derived its name from the shape of the materials dispersion relation, which resembles a hyperbola. This behaviour can also be seen in the canted antiferromagnet studied here as shown in Fig. 7. In each plot, we show both the equifrequency curves for air (incident medium) and $\mathrm{MnF}_{2}$ (refractive medium).

The vectors from the origin to a point on one of the surfaces specifies the direction of the phase propagation in the corresponding plane wave with different wavenumbers $\mathbf{k}$ of the kind $e^{i[\mathbf{k} \cdot \mathbf{r}-\omega(\mathbf{k}) t]}$. In this case the wavepacket has a group velocity [29]

$$
\mathbf{v}_{\mathbf{g}}=\nabla_{k} \omega(\mathbf{k}),
$$

where the gradient is given in $\mathbf{k}$ space of the frequency $\omega=\omega(\mathbf{k})$. The direction of $\mathbf{v}_{\mathbf{g}}$ gives the direction of the propagating wavepacket and in anisotropic materials this generally differs from the direction of the wavevecor $\mathbf{k}$. The vector $\mathbf{v}_{\mathbf{g}}$ is always normal to the equifrequency surface, at $\omega=\omega(\mathbf{k})=$ constant and so is the Poyting vector [30, 31, 32].

In the present case, however, when an external field $\mathbf{B}_{0}$ is applied the equifrequency curve becomes flatter than the equivalent zero field curve, as 

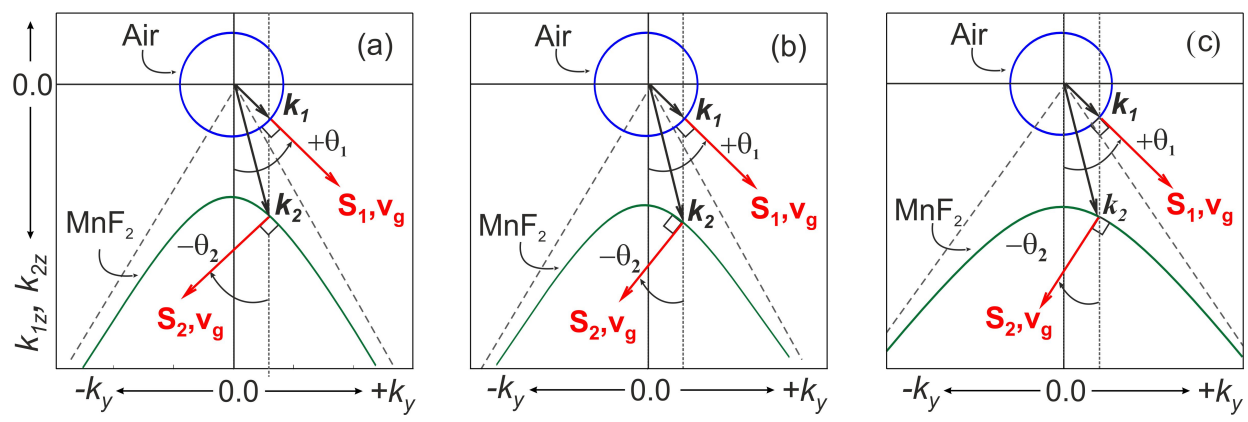

Figure 7: Real parts of the wave-vector component $k_{1 z}$ (blue lines) and $k_{2 z}$ (green lines) as a function of $k_{y}$ (expressed in units of $k_{0}$ ), for transmission in a $\mathrm{MnF}_{2}$ crystal having its extraordinary axis directed along $\mathrm{x}$, in s-polarisation at a frequency $\omega / 2 \pi c=8.984 \mathrm{~cm}^{-1}$. (a) $B_{0}=0.0$, (b) $B_{0}=0.2 \mathrm{~T}$ and (c) $B_{0}=$ $0.4 \mathrm{~T}$.

shown in Figs. 7(b)-(d). This is due to changes in the permeability tensor component $\mu_{z z}$. From these figures, it can be inferred that the effect of the small fields is simply to decrease the magnitude of the angle of refraction $\theta_{2}$ without changing the frequency $\omega$.

The hyperbolic plots shown in Fig. 7, indicate that there is negative refraction of all angles. This is the necessary condition to achieve focusing in flat lenses. This is shown in the diagram of Fig. 8(a). Even though the focusing in Fig. 8(a) seems fairly perfect, in a hyperbolic medium, the focusing of peripheral rays occurs at positions which cannot be described by the paraxial approximation. Marginal rays are focused after the paraxial focus within the slab, and before the paraxial focus outside the slab. This leads to an envelope of interfering refracted rays, also known as caustics.

In this sense, we look at the focusing of the power flow going through an antiferromagnetic flat lens. For this reason, a source radiating in all directions in the $y z$ plane must be used. In Fig. $8(\mathrm{~b})$ the behaviour of the power flow can be seen in great detail as it crosses trhough the lens. As previously discussed, the power flow is given by the time-average Poynting vector $\left\langle\mathbf{S}_{2}\right\rangle$. Due to high levels of transmission for all incident angles, intersecting rays produces a network of constructive and destructive interference within the caustic envelope, both inside and outside the slab. The network of intensities discussed here are examples of caustic curves analogous to those discussed in the classic works by Nye [33]. These caustics are a result of the non-perfect focusing due to hyperbolic behaviour of antiferromagnets. The shape of these caustics can be modified by changing the magnitude of the external applied field $\mathbf{B}_{\mathbf{0}}$. Nevertheless, despite the complications of the 
(a)

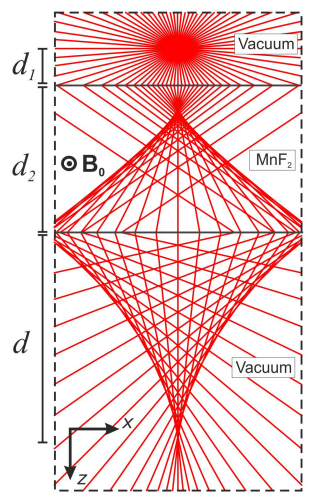

(b)

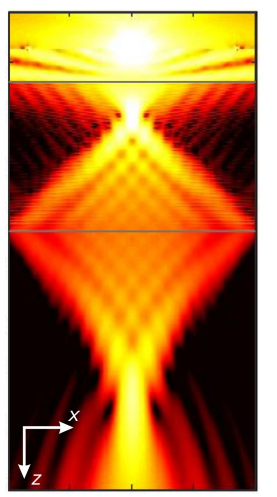

(c)

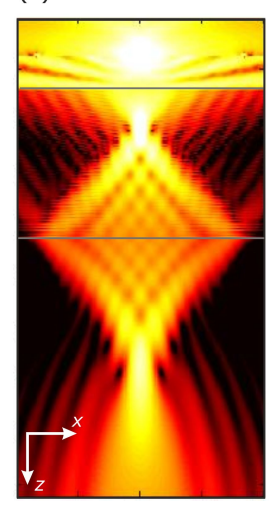

(d)

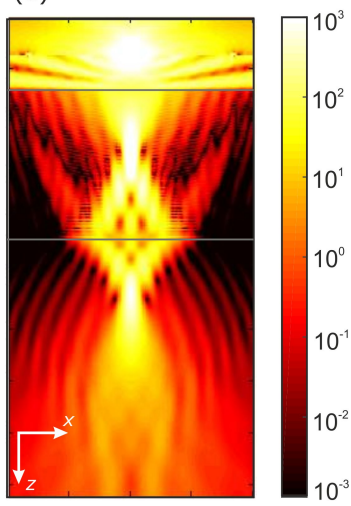

Figure 8: Image formation due to a TE-polarised line source placed above a $\mathrm{MnF}_{2}$ slab. The distance $d_{2}$ is $20 \lambda^{\prime}(\approx 1.0 \mathrm{~cm})$, where $\lambda^{\prime}$ represents the wavelength within the antiferromagnet at normal incidence (about half the free-space wavelength). The distance $d_{1}$ is equal to $d_{2} / 4(\approx 0.25 \mathrm{~cm})$. The extraordinary axis is directed along $x$ and the frequency of the incident radiation satisfies $\omega / 2 \pi c=8.984 \mathrm{~cm}^{-1}$. (a) Schematic showing the path of multiple rays passing through an $\mathrm{MnF}_{2}$ slab for $B_{0}=0.0$. (b) Power flow intensity for the setup shown in (a). Effect of a magnetic field on the intensity profile for (c) $B_{0}=0.2 \mathrm{~T}$ and (d) $B_{0}=0.4 \mathrm{~T}$.The intensity scale is in arbitrary units. Adapted from R. Macêdo, T. Dumelow, and R. L. Stamps, Tunable Focusing in Natural Hyperbolic Magnetic Media, ACS Photonics 3, 1670 (2016).

imperfect focusing at high angles, there remains a high intensity focus at the cusp. Figures 8(c) and 8(d) show how the image is moved closer to the lens by applying an external field.

\section{Polaritons in Antiferromagentic Multiferroics}

Having extensively discussed the behaviour of waves propagating in canted antiferromagnets due to an externally applied Zeeman field, we now turn to another example system: intrinsically canted multiferroics. The geometry is that shown in Fig. 9 which is very similar to that of a canted antiferromagnet given in Fig. 1. However, in the present case the spins lie perpendicular to the crystal's surface and along $z$. The canting angle $\alpha$ arises from a competition between bilinear Heisenberg exchange, that tends to align the magnetic sublattices antiparallel, and an interaction associated with magnetoelectric coupling. In this case the competition appears as 


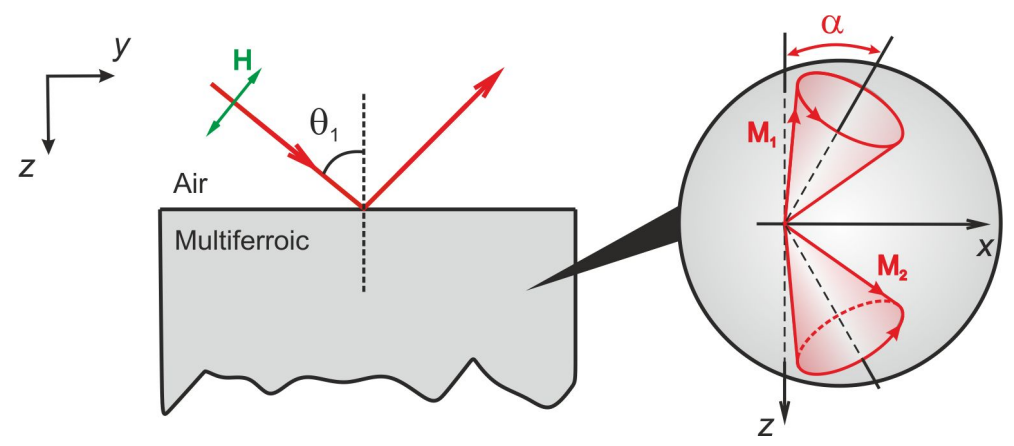

Figure 9: Schematics of an electromagnetic beam incidence at the surface of a multiferroic material as considered throughout this section. The plane of incidence is the $y z$-plane and the radiation is considered to be transverse electric (TE) polarized so that the time-varying $\mathbf{H}$ field of the incident beam lies on the $y z$-plane. The system is spontaneously canted by an angle $\alpha$ towards $x$ as shown in the inset, with electric polarization $\mathbf{P}$ perpendicular to the magnetisation and hence along the $y$ direction. Note that, for clarity only, the horizontal axes in the inset have been flipped and the canting angle has been exaggerated as it is usually very small for this type-material.

an effective field whose magnitude depends on the polarization $\mathbf{P}$. The polarization is affected by static electric fields, temperature, and provides the magneto-electric coupling for time varying electric fields [34]. The canting, is assumed to be given by $[35,36]$

$$
\tan (2 \alpha)=\frac{4 \delta P_{y}}{K+2 \lambda+4 \pi}
$$

where $\delta$ is the magnetoelectric coupling constant, $K$ is a uniaxial anisotropy, $\lambda$ is the Heisenberg exchange, and $P_{y}$ is a spontaneous electric polarisation in the material aligned along the $y$ direction [35]. An example material for which this form of canting is applicable is $\mathrm{BaMnF}_{4}[34,37,38]$. It should be mention that this angle is often quite small, in $\mathrm{BaMnF}_{4}$, for instance, this is only $0.17^{\circ}$.

Similarly to the case of canting due to an external field discussed earlier, the canting angle here is found by energy minimization. No magnetic or electric fields are needed to induce spin canting. However electric fields along the $y$ direction or magnetic fields along the canting direction can affect the resonance frequency.

A phenomenology can be constructed by starting with free energies describing the magnetic, electric and magneto-electric interactions. Details are given in [35], but schematically one includes as a minimum the electric 
energy $F_{E}$, a magnetic contribution $F_{M}$ and a magnetoelectric coupling energy $F_{M E}$ (elastic energies are also typically important but are neglected for simplicity here) [39]. The magnetic susceptibilities are derived as before from the spin equations of motion, but now it is necessary to include the terms describing the magneto-electric coupling. In this case, Eq. 12 for simple spin systems becomes becomes

$$
\frac{1}{\gamma} \frac{d \mathbf{M}}{d t}=\mathbf{M} \times\left[\frac{-\partial}{\partial \mathbf{M}}\left(F_{M}+F_{M E}\right)\right] .
$$

Here, $F_{M}$ is a long wavelength density energy term containing the magnetic contribution and $F_{M E}$ represents an energy density for the magnetoelectric coupling which is assumed to be of the Dzialoshinskii-Moriya form [40].

In this model, it is also necessary to modify the dielectric response in order to accommodate the magneto-electric coupling, and it now becomes

$$
\frac{d^{2} \mathbf{P}}{d t^{2}}=-f \frac{\partial}{\partial \mathbf{P}}\left(F_{M}+F_{M E}\right)
$$

with $F_{E}$ being a fourth order Ginzburg-Landau (G-L) energy density which describes the dielectric contribution through $\mathbf{P}$ and $f$ is the inverse of the phonon mass.

Equations (39) and (40) are used to obtain the dynamic permeability and permittivity tensor components. These assume the same forms as those shown previously. In the present case, the components of $\overleftrightarrow{\mu}(\omega)$, however, assume a very different form from those shown by Eqs. (20)-(19). Their form has been previously derived by Gunawan and Stamps [35], but for simplicity here we continue to work with Eq. (18).

The responses associated with $\overleftrightarrow{\mu}(\omega)$ and $\stackrel{\leftrightarrow}{\varepsilon}(\omega)$ are uncoupled except for certain orientations (i.e. polarization) of the time varying electromagnetic fields with respect to the crystal. For some polarisations and orientations with respect to the crystal there is a magneto-electric response. We consider the case appropriate for $\mathrm{BaMnF}_{4}$, where the magneto-electric response is given by the tensor

$$
\overleftrightarrow{\kappa}(\omega)=\left(\begin{array}{ccc}
0 & \chi_{x y}^{m e} & 0 \\
\chi_{x x}^{m e} & 0 & 0 \\
0 & 0 & 0
\end{array}\right) .
$$

For materials in this class, it is possible to affect the spontaneous electric polarization with a static applied magnetic field, and affect the weak magnetisation with a static electric field. A combination of, and competition among the terms in these tensors determines how electromagnetic waves propagate in multiferroics. This is the subject of the next sections. 


\subsection{Surface Modes and Attenuated Total Reflection}

In order to study the optical properties of multiferroic materials, we use the same approach as in Sec. 2.1. However, due to the magneto-electric coupling the constitutive equations have to be rewritten in order to accommodate the tensor $\overleftrightarrow{\kappa}(\omega)$. Equations (33) and (2) now take the general form:

$$
\mathbf{D}=\stackrel{\leftrightarrow}{\varepsilon}(\omega) \mathbf{E}+4 \pi \overleftrightarrow{\kappa}(\omega) \mathbf{H}
$$

and

$$
\mathbf{B}=\overleftrightarrow{\mu}(\omega) \mathbf{H}+4 \pi \overleftrightarrow{\kappa}(\omega) \mathbf{E}
$$

Because there is no contribution of the magnetoelectric coupling when the incident waves are TE-polarised, these equations and the associated polariton dispersion equations will be the same as those previously derived for simple canted antiferromagnets. However, when considering TE-polirised incident waves, the effect of $\overleftrightarrow{\kappa}(\omega)$ must be accounted for [35]. A full discussion is presented below.

\subsubsection{Trnasverse Electric (TE) Polarisation}

Due the nature of the magnetoelectric coupling, this class of materials has also great potential to display interesting features in their surface polaritons. To investigate this behavior, it is necessary to obtain a dispersion relation assuming solutions for the wave equation in the form of $\mathbf{H} \sim e^{\beta z} e^{j\left(k_{y} y-\omega t\right)}$ for $z<0$ and $\mathbf{H} \sim e^{-\beta_{0} z} e^{j\left(k_{y} y-\omega t\right)}$ for $z>0$. In this case $\beta$ and $\beta_{0}$ are the attenuation constants for the respective media. It is therefore possible to derive a dispersion relation for surface modes which is similar to that of bulk modes. Boundary conditions dictate the continuity of tangential $\mathbf{H}$ and normal $\mathbf{B}$ at the boundary between air and a semi-infinite multiferroic. This gives

$$
\beta_{0}^{2}=k_{y}^{2}-\frac{\omega^{2}}{c^{2}}
$$

and

$$
\mu_{z z} \beta+\left(\mu_{y y} \mu_{z z}-\mu_{y z}^{2}\right) \beta_{0}+\mu_{y z} k_{y}=0,
$$

in vacuum and and in the multiferroic, respectively. 
We now apply this to the example material $\mathrm{BaMnF}_{4}$ where measured values of the weak ferromagnetism [41] are $\mathrm{M}_{C}=18.347 \mathrm{Oe}$, and a canting angle [42] of $\alpha=3 \mathrm{mrad}$ at $\mathrm{T}=4.2 \mathrm{~K}$. These paramaters are used to obtain the magnetization of the sub-latticesusing the relation $M_{c}=2 M_{s} \sin \alpha$, this yields $\mathrm{M}_{s}=3.054 \times 103$ Oe. The approximations by Holmes [43] gives the exchange field, $\mathrm{H}_{E}=50 \mathrm{~T}$, and from the relation $H_{E}=\lambda M_{s}$ we obtain the exchange constant as $\lambda=163.72 \mathrm{erg} \mathrm{cm}^{-3} \mathrm{Oe}^{-2}$. Further parameters for this material have also been provided by Gunawan and Stamps [35, 36].

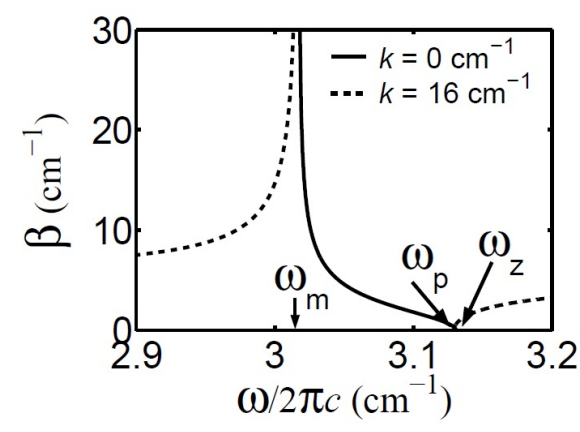

(a)Attenuation constant

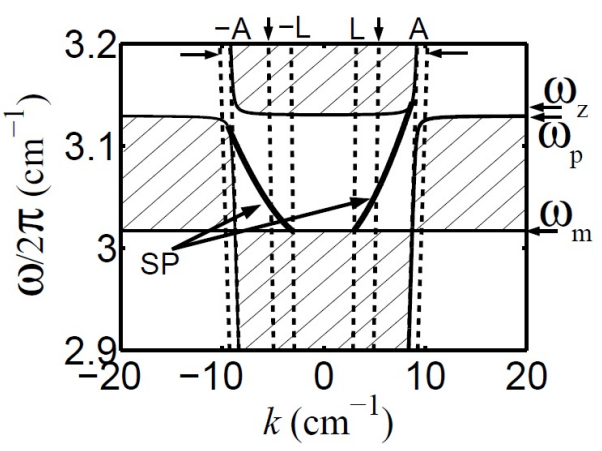

(b)Dispersion relation.

Figure 10: Attenuation constant and dispersion relation. In (a) the attenuation constant is shown for two values of wavevector in the absence of external fields. The solid line represents $\mathrm{k}=0 \mathrm{~cm}^{-1}$, while the dashed line correspond to $\mathrm{k}=16 \mathrm{~cm}^{-1}$. In (b) bulk and surface mode dispersions are shown. Surface modes are indicated by SP. The shaded regions represent bulk bands. The vertical thin lines denoted by vertical and horizontal arrows represent ATR light lines for incident angles of $30^{\circ}$ and $70^{\circ}$. The asymptotic boundaries and lightline are represented by vertical thin lines which are indicated as A and L respectively. Adapted from V. Gunawan and R. L. Stamps, Surface and bulk polaritons in a PML-type magnetoelectric multiferroic with canted spins: TE and TM polarization, Journal of Physics: Condensed Matter 23, 10 (2011).

A plot of both bulk and surface modes is shown in Fig. 10 for $\mathrm{BaMnF}_{4}$. The bulk regions are depicted as shaded regions while the surface modes are shown as sharp lines which only appear where propagation is forbidden. Note that the bulk bands, similarly to simple antiferromagnts, display reciprocal behaviour. However, because of the chosen geometry, in which the magnetisation lies perpendicular to the surface of the crystal, there is a large reststrahl area between the lower and upper bulk band. This is 
because, in this situation, the magnetic field of light couples with the two permeability tensor of opposing sign, $\mu_{x x}$ and $\mu_{z z}$. Note that the propagation of the surface modes is nonreciprocal as can be seen from the differences in position for the sharp $+k$ and $-k$ peaks.

Because these surface modes lie outside the vacuum's light line, they are not strongly coupled to polaritons propagating along the light line in the material. There are different techniques used to excite and study surface modes, including grating couplers and index matching $[2,44,45]$. We discuss here the method of Attenuated Total Reflection (ATR). A schematics of this technique is given in Fig. 11. This technique has been used to detect surface modes at $\mathrm{THz}$ frequencies in a variety of systems including antiferromagnets [2]. It is particularly useful for exciting surface excitations and uses a high optical index prism. Evanescent fields produced by total internal reflection of electromagnetic radiation can couple to surface excitations on an adjacent material whose frequencies are away from the vacuum light line. Surface modes appear in the ATR reflection as sharp dips of reflectivity at certain angles of incidence [44]. An example is given below for the model multiferroic discussed above.

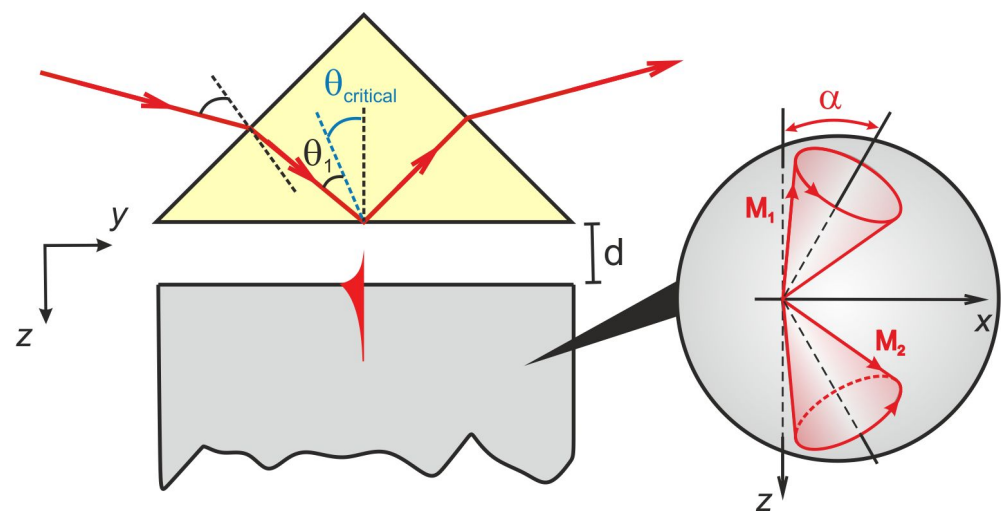

Figure 11: Schematics of classic attenuated total reflection (ATR) experiments considering the multiferroic geometry depicted in Fig. 9. Here. $d$ is the distance between the multiferroic crystal and the dielectric prism.

In order to calculate the ATR reflection, we derive the reflection coefficients taking into account $\beta$ and $\beta_{0}$. Doing this we arrive at

$$
R=\left|\frac{k_{z}\left(1+r e^{-2 \beta_{0} d}\right)-i \beta_{0}\left(1-r e^{-2 \beta_{0} d}\right)}{k_{z}\left(1+r e^{-2 \beta_{0} d}\right)+i \beta_{0}\left(1-r e^{-2 \beta_{0} d}\right)}\right|^{2},
$$

where $d$ is the distance between the prism and the sample as defined in Fig. 11. The reflection coefficient is 


$$
\begin{gathered}
r=\frac{\beta_{0}-\kappa}{\beta_{0}+\kappa}, \\
\kappa=\frac{\mu_{z z} \beta-\mu_{y z} k_{y}}{\mu_{y y} \mu_{z z}-\mu_{y z}^{2}}
\end{gathered}
$$

where the wave vector components of the incident beam are

$$
k_{y}=\varepsilon_{p}^{1 / 2} \frac{\omega}{c} \sin \theta_{1}
$$

and

$$
k_{z}=\varepsilon_{p}^{1 / 2} \frac{\omega}{c} \cos \theta_{1}
$$

where $\varepsilon_{p}$ is the dielectric constant of the prism and $\theta_{1}$ is the incident angle.

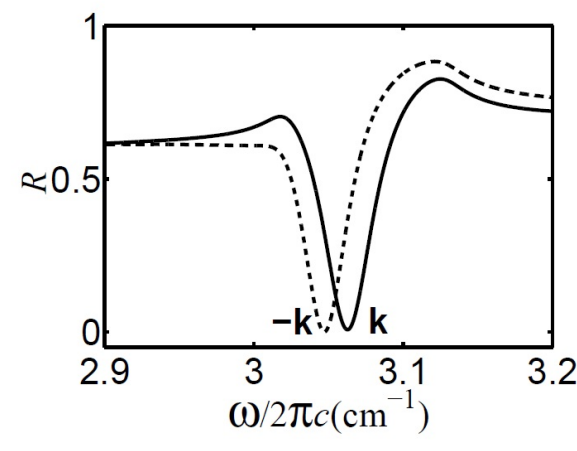

(a)ATR spectra at $30^{\circ}$.

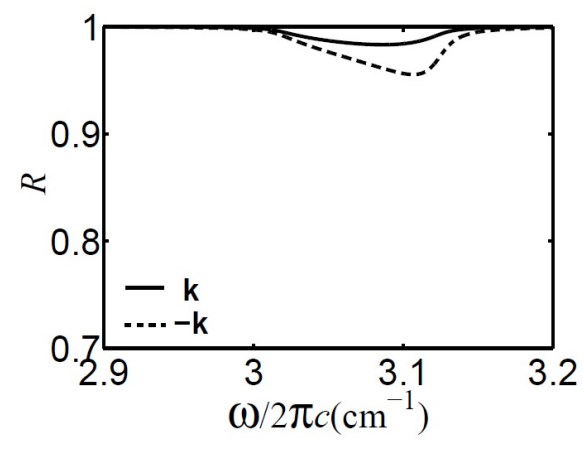

(b)ATR spectra at $70^{\circ}$.

Figure 12: ATR spectra with incident angles (a) $30^{\circ}$ and (b) $70^{\circ}$. In (a) two different sharp dips illustrate the non-reciprocity of the surface modes. In (b) the absence of the sharp dips indicate the absence of surface modes. Adapted from V. Gunawan and R. L. Stamps, Surface and bulk polaritons in a PML-type magnetoelectric multiferroic with canted spins: TE and TM polarization, Journal of Physics: Condensed Matter 23, 10 (2011).

The resulting plots for ATR are given in Fig. 12 for two different incident angles. Part (a) shows $\theta_{1}=30^{\circ}$ and part (b) is for $\theta_{1}=70^{\circ}$. The first plot is a clear example of nonreciprocal surface modes as seen by the reflectivity dips at different frequencies when the incident beam direction is reversed. The second plot however, does not show examples of coupling with surface waves. The dips on these plots are instead associated with bulk polariton modes [35]. By way of comment, we note that in work by Barnas for thin 
film multiferroics, bulk modes can be non-reciprocal for certain propagation directions [34, 37].

\subsubsection{Transverse Magnetic (TM) Polarisation}

In much the same as we have done for TE polarised waves, the propagation of TM polarised waves can be described by solving Maxwell's equations given by Eqs. (23b) and (23d). However, in this plane of polarisation, the magnetic dynamics are coupled to the electric dynamics through the magnetoelectric susceptibilities $\chi^{m e}$ and $\chi^{e m}$.

If we then assume plane waves for $E$ and $H \sim e^{j\left(k_{y} y+k_{z} z \omega t\right)}$ for bulk travelling modes, Maxwell equations provide the following bulk mode dispersion:

$$
\varepsilon_{y y}\left[\varepsilon_{z z} \mu_{x x}\left(\frac{\omega}{c}\right)^{2}-k_{y}^{2}\right]-\varepsilon_{z z}\left(4 \pi \chi^{m e} \frac{\omega}{c}\right)^{2}=0 .
$$

Note that differently from the case of TE polarised waves, here the dispersion relation depends very little on the components of $\overleftrightarrow{\mu}(\omega)$, very much on the components of $\stackrel{\leftrightarrow}{\varepsilon}(\omega)$ and mostly importantly has a contribution of $\overleftrightarrow{\kappa}(\omega)$. We can also use the definition of the surface localised plane wave given by $\mathbf{H} \sim e^{\beta z} e^{i\left(k_{y} y-\omega t\right)}$ for $z<0$ and $\mathbf{H} \sim e^{-\beta_{0} z} e^{i\left(k_{y} y-\omega t\right)}$ for $z>0$ to find the implicit relation for the attenuation constant in the material which is given by

$$
\varepsilon_{y z z}\left(\beta+j 4 \pi \chi^{m e} \frac{\omega}{c}\right)^{2}=\varepsilon_{y y} k_{y}^{2}-\varepsilon_{y y} \varepsilon_{z z} \mu_{x x}\left(\frac{\omega}{c}\right)^{2} .
$$

It is also possible to find an equation for the surface modes. This is done by noting the continuity of the tangential $\mathbf{H}$, tangential $\mathbf{E}$ and normal $\mathbf{D}$ fields. When satisfied, it is possible to obtain the following equation for the dispersion relation:

$$
\left(\beta+j 4 \pi \chi^{m e} \frac{\omega}{c}\right)+\varepsilon_{y y} \beta_{0}=0 .
$$

Solutions for the TM bulk and surface modes are shown in Fig. 13. Two gaps can be seen in the bulk regions shown in part (a). These are created by the electric and magnetic contributions in which case the gap around $3 \mathrm{~cm}^{-1}$ is of the same nature as that shown for TE polarised waves. The gap around $41 \mathrm{~cm}^{-1}$, on the other hand, is due to the magneto-electric interaction. This gap, shown in part (b) is strongly dependent on the components of $\overleftrightarrow{\kappa}(\omega)$, disappearing if terms are zero. The gap becomes wider if the coupling 

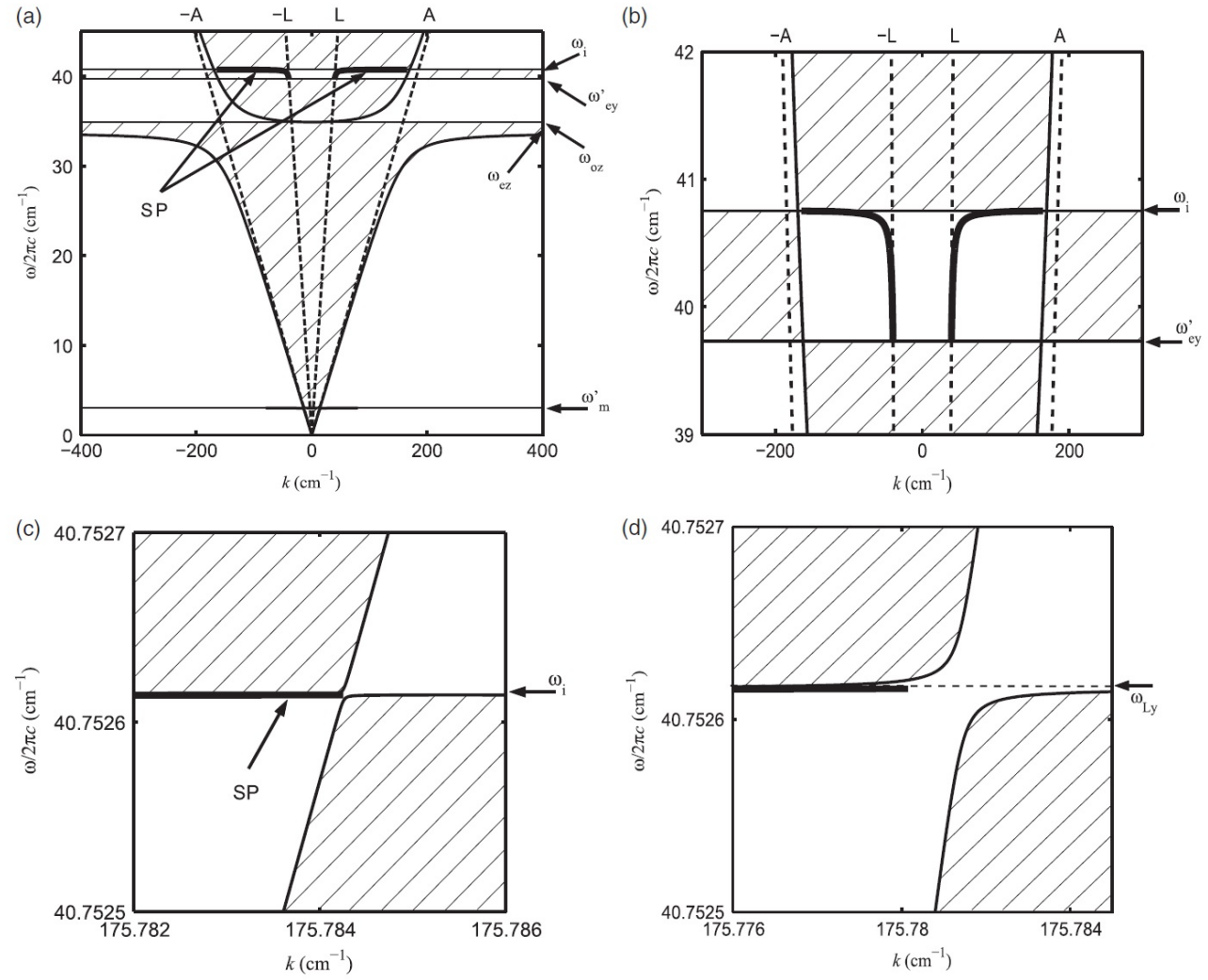

Figure 13: (a) The dispersion relation without the external field is shown. The surface modes are indicated by 'SP'. The shaded regions represent bulk bands. (b) The 'window' where the surface modes exist is shown. (c) A narrow gap around $\omega_{i}$ is expanded. (d) The narrow gap is wider when the ME coupling is increased by a factor of 10 . Adapted from V. Gunawan and R. L. Stamps, Surface and bulk polaritons in a PML-type magnetoelectric multiferroic with canted spins: TE and TM polarization, Journal of Physics: Condensed Matter 23, 10 (2011).

strength increases and this shown in part (d) for the case where the coupling is increased by a factor of ten when compared to that shown in part (c).

The gap at which propagation is not allowed allows for the possible existence of an interesting class of surface localised excitations. This class of excitation is characterised by a complex attenuation and requires dissipation to be included in the theoretical model.

In Fig. 14 we show the resulting curves for the attenuation constant given by Eqs. 52 and 53 . These curves are of particular interest since, differently from those for TE polarised surface modes, it is only possible to satisfy the 

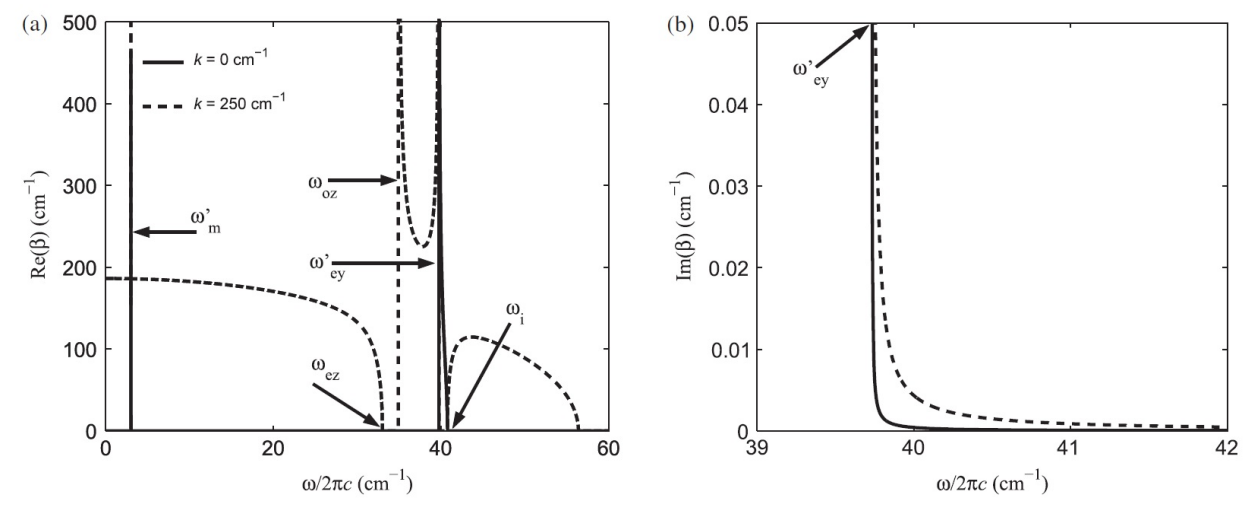

Figure 14: Attenuation constant as a function of frequency. (a) The real part of the attenuation constant is shown for two values of wavevector in the absence of any external fields. The solid line represents $\mathrm{k}=0 \mathrm{~cm}^{-1}$, while the dashed line corresponds to $\mathrm{k}=250 \mathrm{~cm}^{-1}$. (b) The imaginary part of the attenuation constant is shown for two values of ME coupling. The solid line is for $1.42 \times 10^{-5} \mathrm{~cm}^{2} / \mathrm{statC}$, and the dashed line represents the coupling = $1.42 \times 10^{-4} \mathrm{~cm}^{2} /$. Adapted from V. Gunawan and R. L. Stamps, Surface and bulk polaritons in a PML-type magnetoelectric multiferroic with canted spins: TE and TM polarization, Journal of Physics: Condensed Matter 23, 10 (2011).

boundary conditions with a complex $\beta$. This is known as a pseudo-surface mode rather than a true surface mode. These are surface modes in which the energy is not purely localised to the surface and instead, the energy 'leaks' into the bulk. The real part $\beta$ determines how the mode travels along the surface and decays into the material, whereas the imaginary part $\beta$ gives the wave number at which the wave travels into the medium.

\section{Summary and Outlook}

We have discussed how electromagnetic waves can be manipulated by spin canted media. This can happen in the form of nonreciprocal reflection, surface modes, lateral shifts on reflection, and negative refraction of the refracted beam.

In simple antiferromagnetic crystals, canting can be induced by an applying an external magnetic field perpendicular to the spin orientation direction. This leads to non-reciprocal reflection coefficients and non-reciprocal GoosHänchen. Moreover, the orientation of the spin system leads to hyperbolic 
dispersion and large regions of propagation close to the magnon-polariton resonance. This can be used to obtain negative refraction and field tunable focusing in flat lenses.

In multiferroics, the effects of nonreciprocity as somewhat similar to that of simple field canted antiferromagnts. In this case however, there is interest in propagation of both TE and TM polarized waves due to the nature of the coupled excitations in these media. The non-reciprocal reflections are largely affected by the surface modes in both planes of polarisation.
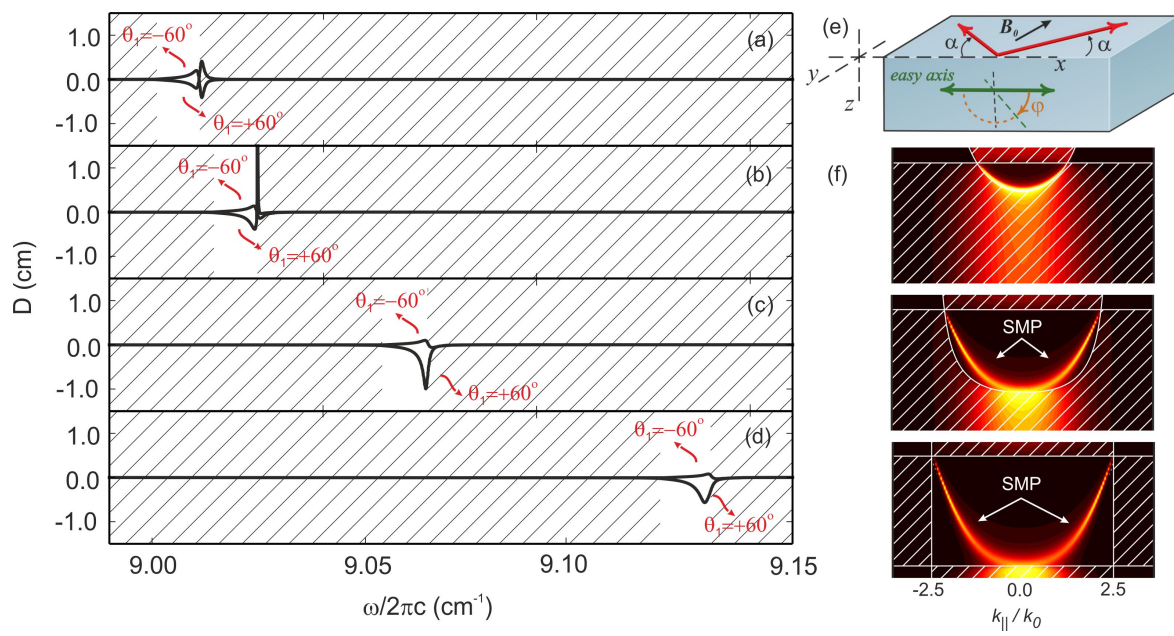

Figure 15: Goos-Hänchen shift, $\mathrm{D}$, for a beam reflected at the surface of $\mathrm{MnF}_{2}$ with $\theta_{1}= \pm 60^{\circ}$ and different values of applied external field (a) $\mathrm{B}_{0}=0.0 \mathrm{~T}$, (b) $\mathrm{B}_{0}=0.5 \mathrm{~T}$, (c) $\mathrm{B}_{0}=1.0 \mathrm{~T}$ and (d) $\mathrm{B}_{0}=1.5 \mathrm{~T}$. (e) Geometry of anisotropy rotation in a canted antiferromagnet due an externally applied field and (f) the effect of rotation on surface magnon-polaritons probed through ATR. Based on data from R. Macêdo, R. L. Stamps, and T. Dumelow, Spin canting induced nonreciprocal Goos-Hüchen shifts, Opt. Express 22, 28467 (2014) and R. Macêdo and R. E. Camley, Engineering terahertz surface magnon-polaritons in hyperbolic antiferromagnets, Phys. Rev. B 99, 014437 (2019).

For field canted antiferromagnets we considered the spins to lie parallel to the surface of the crystal; and for multiferroics we considered the spins to lie perpendicular to the surface. In the first example we discussed reflection and transmission, and in the second example we concentrated on surface localised excitations. These two cases describe only two of many possibilities.

To illustrate this point, in Fig. 15(a)-(d) we show the effect of various externally applied magnetic field on the Goos-Hänchen shifts. The figure shows how the applied field shifts the resonance to higher frequencies and 
how the displacement becomes nonreciprocal. The effect of the field on the displacement is distinctly nonlinear. This has been pointed out to be associated with coupling of the incident radiation to surface resonances [15]. For instance, the reflectivity dip in Fig. 4 at negative $\theta_{1}$ is actually an example of coupling to a surface resonance. This implies an increased penetration into the antiferromagnet, so it is reasonable to expect a higher absorption [4]. In this sense, surface modes in multiferroics, could be used to exploit the features of possible lateral shifts on the reflection of radiation from these surfaces. These surface waves are highly dependent on the anisotropy direction, therefore by controlling this it is possible to control and widen the regions where these waves appear. In Fig. 15(e) an example geometry is shown of how the axis can be rotated by an angle $\varphi$ with respect to the surface. By doing so, it is possible to open up gaps of non-propagating regions where surface polaritons can appear as shown in Fig. 15(f) [46]. As demonstrated recently, this rotation allows for the existence of surface waves even at zero external field which could be used as a way to enhance Goos-Hänchen shifts.
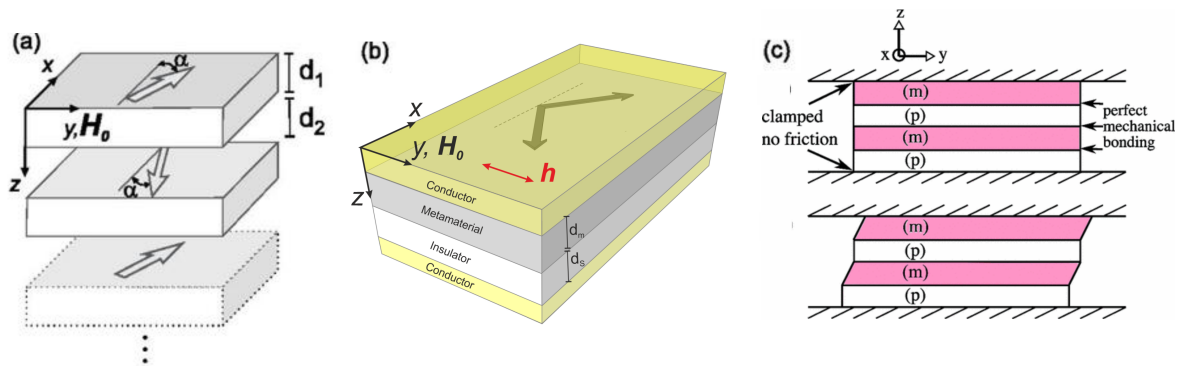

Figure 16: Artificial canted systems for devices: We show examples for (a) an artificial canted multilayer structure, (b) a waveguide micro-strip device for signal processing based on the multilayer shown in (a) and in (c) we show examples of how strain can affect heterostructures with magnetoelectic coupling. Based on data from R. Macêdo, K. L. Livesey and R. E. Camley, Using magnetic hyperbolic metamaterials as high frequency tunable filters, Appl. Phys. Lett. 113, 121104 (2018) and K. L. Livesey, Strain-mediated magnetoelectric coupling in magnetostrictive/piezoelectric heterostructures and resulting highfrequency effects, Phys. Rev. B 83, 224420 (2011).

Throughout this chapter, we have discussed natural canted systems which work mostly at low temperatures. This is often a challenge to realise experiments and limits their applicability. However, it is possible to engineer artificial canted structures that emulate the behavior of natural crystals whilst working at room temperatures. For example, in Fig. 16(a) we show 
a multilayer structures comprising magnetic layers separated by a nonmagnetic spacer layer [47]. By controlling the thickness of the spacer layer it is possible to induce antiferromagnetic coupling, and therefore generate spin canting by means of an externally applied field. These structure can be used to achieve resonances in the high $\mathrm{GHz}$ range, and can be easily incorporated into signal processing and communication devices such as ban-pass or band-stop filters. An example of such structure, in the form of a micro-strip waveguide, is shown in Fig. 16(b).

In the case of multiferroics, their performance can also be enhance, or magnetoelectric coupling can be built into heterostructures, such as the one shown in Fig. 16(c). However, as studied by Livesey, strain between the layers may affect the strength of the magnetoelectric coupling and therefore affect their resonance frequencies [48]. It is important point out here that if the geometry of the multiferroic is chosen as to imitate that chosen for antiferromagnetic crystals, hyperbolic behaviour and hence negative refraction of all angles should also be expected. However, in multiferroic crystals, both lateral shifts on reflection and negative refraction would be affect not only by an externally applied magnetic field but also by externally applied electric fields.

\section{Acknowledgements}

The authors would like to thank Gavin M. Macauley for a careful reading of the chapter and for helpful comments. We would also like to thank Karen L. Livesey for comments and discussions. The work of Rair Macêdo was supported by the Leverhulme Trust and the University of Glasgow through LKAS funds and Robert L. Stamps acknowledges the support of the Natural Sciences and Engineering Research Council of Canada (NSERC RGPIN 05011-18).

\section{References}

1. Keqian Zhang and Dejie Li. Chapter 8 Electromagnetic Waves in Anisotropic Media, pages 453-534. Springer Berlin Heidelberg, Berlin, Heidelberg, 1998.

2. Robert E. Camley. Nonreciprocal surface waves. Surf. Sci. Rep., 7(34):103-188, jul 1987.

3. C. Kittel. Theory of antiferromagnetic resonance. Phys. Rev., 82:565-565, May 1951. 
4. F. Lima, T. Dumelow, E. L. Albuquerque, and J. A. P. da Costa. Nonreciprocity in the Goos-Hänchen shift on oblique incidence reflection off antiferromagnets. J. Opt. Soc. Am. B, 28(2):306-313, Feb 2011.

5. title=Canted Antiferromagnetism: Hematite Morrish, A H. World Scientific, 1995.

6. Charles Kittel. Introduction to Solid State Physics. John Wiley \& Sons, Inc., New York, 6th edition, 1986.

7. A. G. Gurevich, E. I. Golovenchits, and V. A. Sanina. Antiferromagnetic resonance and origin of canting in orthorhombic crystals. Journal of Applied Physics, 39(2):1023-1024, 1968.

8. A. M. Kadigrobov N. I. Gordon and M. A. Savchenko. Zh. Eksperim. Teor. Fiz, 48(864), 1965 [English transl.: N. I. Gordon, A. M. Kadigrobov, and M. A. Savchenko, Soviet Phys.- JETP 21, 576 (1965)].

9. I.E. Dzialoshinskii. Thermodynamic theory of weak ferromagnetism in antiferromagnetic substances. Zh. Eksperim. Teor. Fiz, 32(1547-1562), 1957; [JETP 5, 1259-1272 (1957)].

10. D. L. Mills and E. Burstein. Polaritons: the electromagnetic modes of media. Rep. Prog. Phys., 37(7):817-926, jul 1974.

11. R. E. Camley and D. L. Mills. Surface polaritons on uniaxial antiferromagnets. Phys. Rev. B, 26:1280-1287, 1982.

12. R. L. Stamps, R. E. Camley, F. C. Nörtemann, and D. R. Tilley. Dynamic susceptibilities for magnetic layered structures. Phys. Rev. B, 48:1574015743, Dec 1993.

13. Takeo Nagamiya. Theory of antiferromagnetism and antiferromagnetic resonance absorption, ii. Progress of Theoretical Physics, 6(3):350-355, 1951.

14. R. Macêdo and T. Dumelow. Tunable all-angle negative refraction using antiferromagnets. Phys. Rev. B, 89:035135, Jan 2014.

15. R. Macêdo, R. L. Stamps, and T. Dumelow. Spin canting induced nonreciprocal goos-hänchen shifts. Opt. Express, 22:28467, Nov 2014.

16. N. S. Almeida and D. L. Mills. Dynamical response of antiferromagnets in an oblique magnetic field: Application to surface magnons. Phys. Rev. B, 37(7):3400-3408, Mar 1988.

17. Thomas Dumelow and Marcílio C. Oliveros. Continuum model of confined magnon polaritons in superlattices of antiferromagnets. Phys. Rev. B, 55(2):994-1005, jan 1997. 
18. R. L. Stamps, B. L. Johnson, and Robert E. Camley. Nonreciprocal reflection from semi-infinite antiferromagnets. Phys. Rev. B, 43(4):36263636, feb 1991.

19. L. Remer, E. Mohler, W. Grill, and B. Lüthi. Nonreciprocity in the optical reflection of magnetoplasmas. Phys. Rev. B, 30(6):3277-3282, sep 1984.

20. R. Macêdo, R. Rodrigues da Silva, T. Dumelow, and J. A. P. da Costa. $\mathrm{MgF}_{2}$ as a material exhibiting all-angle negative refraction and subwavelength imaging due to the phonon response in the far infrared. Optics Communications, 310(0):94 - 99, 2014.

21. $R$ Macêdo and $T$ Dumelow. Beam shifts on reflection of electromagnetic radiation off anisotropic crystals at optic phonon frequencies. Journal of Optics, 15(1):014013, 2013.

22. Rémi H. Renard. Total reflection: a new evaluation of the Goos-Hänchen shift. J. Opt. Soc. Am., 54(10):1190-1197, oct 1964.

23. Rair Macêdo, Thomas Dumelow, and Robert L. Stamps. Tunable focusing in natural hyperbolic magnetic media. ACS Photonics, 3(9):1670-1677, 2016.

24. Thomas Dumelow and David R. Tilley. Optical properties of semiconductor superlattices in the far infrared. J. Opt. Soc. Am. A, 10:633-645, apr 1993.

25. Rair Macêdo. Chapter two - tunable hyperbolic media: Magnonpolaritons in canted antiferromagnets. volume 68 of Solid State Physics, pages 91 - 155. Academic Press, 2017.

26. Anthony J. Hoffman, Leonid Alekseyev, Scott S. Howard, Kale J. Franz, Dan Wasserman, Viktor A. Podolskiy, Evgenii E. Narimanov, Deborah L. Sivco, and Claire Gmachl. Negative refraction in semiconductor metamaterials. Nature Materials, 6(12):946-950, DEC 2007.

27. R. Rodrigues da Silva, R. Macêdo da Silva, T. Dumelow, J. A. P. da Costa, S. B. Honorato, and A. P. Ayala. Using phonon resonances as a route to all-angle negative refraction in the far-infrared region: The case of crystal quartz. Phys. Rev. Lett., 105(16):163903, Oct 2010.

28. R. Estevâm da Silva, R. Macêdo, T. Dumelow, J. A. P. da Costa, S. B. Honorato, and A. P. Ayala. Far-infrared slab lensing and subwavelength imaging in crystal quartz. Phys. Rev. B, 86:155152, Oct 2012.

29. Nathan Marcuvitz Leopold B. Felsen. Radiation and Scattering of Waves. Wiley-IEEE Press, New Jersey, 1973.

30. Lorenzo Ferrari, Chihhui Wu, Dominic Lepage, Xiang Zhang, and Zhaowei Liu. Hyperbolic metamaterials and their applications. Progress in Quantum Electronics, 40:1 - 40, 2015. 
31. Anan Fang, Thomas Koschny, and Costas M. Soukoulis. Optical anisotropic metamaterials: Negative refraction and focusing. Phys. Rev. B, 79:245127, Jun 2009.

32. Karolina Korzeb, Marcin Gajc, and Dorota Anna Pawlak. Compendium of natural hyperbolic materials. Opt. Express, 23(20):25406-25424, Oct 2015.

33. J F Nye. Natural Focusing and Fine Structure of Light: Caustics and Wave Dislocations. Institute of Physics Publishing: Bristol and Philadelphia, 1999.

34. J. Barnaś. Electromagnetic modes in magnetic systems with linear magneto-electric interactions. Journal of Magnetism and Magnetic Materials, 62(2):381 - 391, 1986.

35. V Gunawan and R L Stamps. Surface and bulk polaritons in a pml-type magnetoelectric multiferroic with canted spins: Te and tm polarization. Journal of Physics: Condensed Matter, 23(10):105901, 2011.

36. Vincensius Gunawan and Robert L Stamps. Mixed modes of surface polaritons in a pml-type magnetoelectric multiferroic with canted spins. Journal of Physics: Condensed Matter, 24(40):406003, 2012.

37. J Barnas. Polaritons in ferromagnets with linear magneto-electric properties. Journal of Physics C: Solid State Physics, 19(3):419, 1986.

38. D. R. Tilley and J. F. Scott. Frequency dependence of magnetoelectric

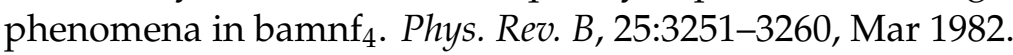

39. V. D. Buchel'nikov and V. G. Shavrov. New types of surface waves in magnetoelectric antiferromagnets. JETP, 109(2):308, 1996.

40. Claude Ederer and Craig J Fennie. Electric-field switchable magnetization via the dzyaloshinskii-moriya interaction: Fetio 3 versus bifeo 3. Journal of Physics: Condensed Matter, 20(43):434219, 2008.

41. J F Scott. Phase transitions in bamnf 4. Reports on Progress in Physics, 42(6):1055, 1979.

42. E. L. Venturini and F. R. Morgenthaler. Afmr versus orientation in weakly ferromagnetic bamnf4. AIP Conference Proceedings, 24(1):168-169, 1975.

43. L. Holmes, M. Eibschütz, and H.J. Guggenheim. Spin-flop transition in bamnf4. Solid State Communications, 7(14):973 - 976, 1969.

44. K Abraha, S R P Smith, and D R Tilley. Surface polaritons and attenuated total reflection spectra of layered antiferromagnets in the faraday configuration. Journal of Physics: Condensed Matter, 7(32):6423, 1995. 
45. Kamsul Abraha and David R. Tilley. Theory of far infrared properties of magnetic surfaces, films and superlattices. Surf. Sci. Rep., 24(5-6):129-222, 1996.

46. Rair Macêdo and Robert E. Camley. Engineering terahertz surface magnon-polaritons in hyperbolic antiferromagnets. Phys. Rev. B, 99:014437, Jan 2019.

47. Rair Macêdo, Karen L Livesey, and Robert E Camley. Using magnetic hyperbolic metamaterials as high frequency tunable filters. Applied Physics Letters, 113(12):121104, 2018.

48. K. L. Livesey. Strain-mediated magnetoelectric coupling in magnetostrictive/piezoelectric heterostructures and resulting high-frequency effects. Phys. Rev. B, 83:224420, Jun 2011. 